\title{
Maximum Tolerated Dose Evaluation of the AMPA Modulator Org 26576 in Healthy Volunteers and Depressed Patients
}

\section{A Summary and Method Analysis of Bridging Research in Support of Phase II Dose Selection}

Kari R. Nations, ${ }^{1}$ Roberta Bursi, ${ }^{2}$ Peter Dogterom, ${ }^{2}$ Larry Ereshefsky, ${ }^{3,4}$ Lev Gertsik, ${ }^{3}$ Tim Mant ${ }^{5}$ and Jacques Schipper ${ }^{2}$

1 Merck Sharp \& Dohme Corp., Whitehouse Station, NJ, USA

2 Merck Sharp \& Dohme Oss BV, Oss, the Netherlands

3 California Clinical Trials Medical Group, Inc., Parexel International, Glendale, CA, USA

4 University of Texas Health Science Center, San Antonio, TX, USA

5 Quintiles Drug Research Unit at Guy's Hospital, London, UK

Background: A key challenge to dose selection in early central nervous system (CNS) clinical drug development is that patient tolerability profiles often differ from those of healthy volunteers (HVs), yet HVs are the modal population for determining doses to be investigated in phase II trials. Without clear tolerability data from the target patient population, first efficacy trials may include doses that are either too high or too low, creating undue risk for study participants and the development program overall. Bridging trials address this challenge by carefully investigating safety and tolerability in the target population prior to full-scale proof-of-concept trials.

Objective: Org 26576 is an alpha-amino-3-hydroxy-5-methylisoxazole4-propionic acid (AMPA) receptor positive allosteric modulator that acts by modulating ionotropic AMPA-type glutamate receptors to enhance glutamatergic neurotransmission. In preparation for phase II efficacy trials in major depressive disorder (MDD), two separate phase I trials were conducted to evaluate safety, tolerability, and pharmacokinetics in HVs and in the target patient population.

Methods: Both trials were randomized and placebo controlled, and included multiple rising-dose cohorts (HV range 100-400 mg bid; MDD range $100-600 \mathrm{mg}$ bid). HVs $(\mathrm{n}=36)$ and patients with MDD $(\mathrm{n}=54)$ were dosed under similarly controlled conditions in an inpatient facility, HVs for up to 14 days and MDD patients for up to 28 days. Safety, tolerability, and pharmacokinetics were assessed frequently. 
Results: Despite comparable pharmacokinetic profiles, the maximum tolerated dose (MTD) in depressed patients was $450 \mathrm{mg}$ bid, twice the MTD established in HVs. No clinically relevant safety issues associated with Org 26576 were noted. Conclusion: This article presents safety, tolerability, and pharmacokinetic data from two different populations examined under similar dosing conditions. The important implications of such bridging work in phase II dose selection are discussed, as are study design and data interpretation challenges.

\section{Introduction}

One of the critical challenges in early-stage clinical drug development is the selection of appropriate doses for initial efficacy trials. The lack of validated biomarkers in most central nervous system (CNS) indications leads to phase II dose and regimen selection that is often based on a best guess for efficacy and on safety/tolerability established in preclinical and early phase I work. Although human tolerability is most often determined via early studies in healthy volunteers (HVs), there is good evidence that tolerability profiles in healthy subjects do not necessarily predict tolerability in target patient populations, particularly in CNS disorders. ${ }^{[1]}$ Bridging studies, sometimes known as phase Ib studies, offer a unique opportunity to examine tolerability in target populations in support of dose selection for phase II efficacy trials. Establishing the patient maximum tolerated dose (MTD) as early as possible not only reduces the risk that patients in proofof-concept trials will be over- or under-exposed to study medication, but also can result in acceleration of the drug development timeline. ${ }^{[2]}$ These trials also provide the opportunity to assess preliminary dose and/or pharmacokinetic relationships with pharmacodynamic measures, including electrophysiologic or neurochemical biomarkers, as well as cognitive or behavioral endpoints. ${ }^{[3,4]}$

Much of the published bridging work to date has been conducted in Alzheimer's disease and schizophrenia, where small numbers of otherwise healthy patients are exposed to escalating doses of the study drug under controlled conditions. ${ }^{[5]}$ Although there is variability between trials, the MTD is generally defined as the dose one level (or 'step') below the dose that causes an unacceptable number of discontinuations or dose-limiting adverse events (AEs). ${ }^{[6]}$ Doses included in these bridging trials are often selected on the basis of $\mathrm{HV}$ data, with an expanded range to allow for the possibility that patient and $\mathrm{HV}$ tolerability may differ. Indeed, bridging trials have often led to conclusions that were disparate from those that might have been drawn on the basis of HV data alone. ${ }^{[7-15]}$ Despite relatively comparable pharmacokinetic profiles in most cases, the resulting MTD in these trials was determined to be higher than - and in some cases a multiple of - the MTD in HVs. Importantly, there is no evidence from these trials that safety profiles (i.e. findings on objective safety measures) differ between HVs and patients; the differences appear to be limited to tolerability (i.e. AEs).

The reason for this population-dependent tolerability phenomenon is largely unknown, but it has been suggested that it is due to alteration of receptor activity by prior treatments or by the disease itself. ${ }^{[16]}$ Still, one must exercise caution when drawing generalized conclusions. Although the majority of studies indicate that patients are able to tolerate higher doses than HVs, there are examples where there is no difference or even the opposite is true. ${ }^{[2,17]}$ Furthermore, conflicting outcomes within the same drug class (e.g. acetylcholinesterase inhibitors) ${ }^{[12,17]}$ suggest that specific molecule differences may play a contributory role. Such divergent findings underscore the importance of carefully evaluating tolerability in the target population prior to embarking on phase II efficacy trials of any new investigational drug. While the cumulative MTD literature in schizophrenia and Alzheimer's disease can lend some guidance to drug developers in the CNS arena, published data are comparatively sparse for other 


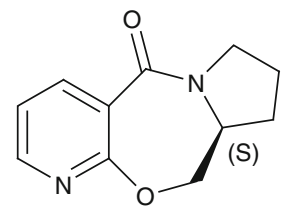

Fig. 1. Chemical structure of Org 26576.

indications, including major depressive disorder (MDD).

The current paper summarizes the bridging data for Org 26576 (chemical name: [9aS]-8,9,9a,10tetrahydro-5H,7H-pyrido[3,2-f]pyrrolo[2,1-c][1,4] oxazepin-5-one; see figure 1). Org 26576 belongs to a novel class of compounds referred to as alphaamino-3-hydroxy-5-methylisoxazole-4-propionic acid receptor positive allosteric modulators (AMPA PAMs), which act by modulating ionotropic AMPA-type glutamate receptors to enhance glutamatergic neurotransmission. ${ }^{[18,19]}$ Dysregulation of the glutamatergic system has been implicated in the pathology of psychiatric diseases such as schizophrenia ${ }^{[20,21]}$ and mood disorders. ${ }^{[22-24]}$ AMPA receptors mediate fast excitatory neurotransmission in the brain, and their activation has been reported to exert a variety of cellular effects, including enhancement of neurotrophic factor activity (particularly brain-derived neurotrophic factor $[\mathrm{BDNF}]),{ }^{[25]}$ synaptic plasticity, ${ }^{[26]}$ and neurogenesis. ${ }^{[27]}$ It has been suggested that modulation of these cellular activities may, in part, play a role in the mode of action of current antidepressant agents. ${ }^{[28,29]}$ If so, AMPA PAMs represent a promising novel approach in MDD.

The two trials presented here were undertaken by employing very similar designs and dosing approaches in order to characterize the tolerability, safety, and pharmacokinetic profiles of Org 26576 both in HVs and in patients diagnosed with MDD. The overarching program objective of these trials was to facilitate dose selection for the first proofof-concept trials with Org 26576 in MDD. HV and patient safety/tolerability and pharmacokinetic data that contributed to dosing decisions are presented here. Secondary, exploratory pharmacodynamic endpoints from the patient trial are presented elsewhere. ${ }^{[30]}$

\section{Methods}

\section{Study Design and Subjects}

The studies described herein were conducted in accordance with the principles of Good Clinical Practice and were approved by each center's institutional review board and the appropriate regulatory agency prior to commencement of study activities. All study participants signed an informed consent form before any screening evaluations were performed. Study objectives of both trials were to assess the safety and tolerability, MTD, pharmacokinetics, and pharmacodynamics of Org 26576.

\section{Study 1: A Combined Single- and Multiple-Oral- Dose Tolerability and Pharmacokinetic Study of Org 26576 in Healthy Male Subjects (Organon Protocol 21301)}

This study was conducted at Guy's Drug Research Unit, Quintiles Ltd, London, UK, between June and November 2005. This was a randomized, double-blind, crossover, placebo-controlled, singlerising-dose study (part I), and a randomized, double-blind, parallel-group, placebo-controlled, multiple-rising-dose study (part II) in healthy male volunteers aged 18 to 45 years. In the singledose part of the study (part I), two groups of nine subjects each participated in three successive periods during which they received a single dose of Org 26576 (range 5-250 mg) on two separate occasions and a single dose of placebo on one occasion. The washout period between successive dosing occasions was at least 7 days.

The multiple-dose part of the study (part II) included two sequential nine-subject groups, where six in each group received Org 26576 and three received placebo. In part II, group 3 subjects received either a single dose of Org 26576 $(100 \mathrm{mg})$ or placebo on 3 of 9 days and twicedaily (bid) doses at 12-hour intervals on days 3-8. In this group, the effect of food (a high-fat breakfast) on the pharmacokinetics of Org 26576 was investigated on day 5; four subjects received the morning dose after a standardized high-fat breakfast, and five subjects received the morning dose after an overnight fast. Subjects received the opposite food regimen on day 6 . Thereafter, no food 
was permitted until 4 hours post-dose. In part II, group 4 utilized a multiple-rising-dose design to determine the MTD. Subjects received either Org 26576 or placebo at 12-hour intervals; starting doses were based on tolerability results from previous groups and were carefully escalated in interim steps of 1.25-1.5 times the previous dose as follows: $100 \mathrm{mg}$ bid on days 1 and $2,150 \mathrm{mg}$ bid on days $3-5,225 \mathrm{mg}$ bid on days $6-8,325 \mathrm{mg}$ bid on days 9-11, $400 \mathrm{mg}$ bid on days 12 and 13, and a single $400 \mathrm{mg}$ dose on day 14 .

\section{Study 2: Multiple-Oral-Dose Tolerability and Pharmacokinetics of Org 26576 in Patients Diagnosed with Major Depressive Disorder (Organon Protocol 174001)}

This study was conducted at California Clinical Trials in Glendale, CA, USA, between September 2007 and December 2008 (clinicaltrials.gov identifier: NCT00610649). Part I was a randomized, double-blind, placebo-controlled, multiple-risingdose evaluation in 24 patients. Four sequential cohorts of six patients each received either Org 26576 or placebo in a 2:1 ratio (cohort A dose titration: $100 \mathrm{mg}$ bid on days $1-3,200 \mathrm{mg}$ bid on days $4-6,300 \mathrm{mg}$ bid on days $7-9,450 \mathrm{mg}$ bid on days $10-12,600 \mathrm{mg}$ bid on days 13-16). Within part I, cohorts A, B, and C started at progressively higher doses (100 mg, $200 \mathrm{mg}$, or $300 \mathrm{mg}$ ), all rising to $600 \mathrm{mg}$ bid, in order to explore the optimal titration schedule; cohort D evaluated cerebrospinal fluid (CSF) pharmacokinetics at the lower end of the dosing range (at 100 and $300 \mathrm{mg}$ bid). The treatment duration in part I was between 10 and 16 days, depending on the titration schedule. Part II was a 30-patient, randomized, doubleblind, parallel-group, placebo-controlled design, which evaluated two dose levels of Org 26576 for 28 days (10 subjects assigned to $100 \mathrm{mg}$ bid, 10 subjects assigned to $400 \mathrm{mg}$ bid, and 10 subjects assigned to placebo), with objectives to evaluate tolerability, pharmacokinetics, and pharmacodynamics over an extended treatment period. The doses in part II were selected on the basis of the tolerability results from part I.

All selected patients were male or female, aged 18-65 years, and diagnosed with MDD according to the fourth edition of the Diagnostic and Sta- tistical Manual of Mental Disorders (DSM-IV-TR). Current depressive episodes were mild to severe without psychotic features, and no more than 2 years in duration, with a total score of at least 9 but not more than 20 on the Quick Inventory of Depression Symptomatology - Clinician Rating (QIDS-C). ${ }^{[31]}$ Patients who had received antidepressant treatment with an adequate dose and duration in the current episode were excluded. Eligible patients were otherwise generally healthy and medically stable; were taking no concurrent psychotropic medications; and had no history of bipolar disorder, psychosis, post-traumatic stress disorder, obsessive-compulsive disorder, or eating disorder. Patients with a 6-month history of substance dependence (not including nicotine), current substance abuse, or a positive screening or admission urine drug/alcohol test were excluded.

Subjects in both trials were admitted to the unit 1-2 days before the first dosing and confined for the full term of the dosing period. Diet and physical activity were controlled, and subjects were closely monitored for safety and tolerability. Safety evaluations included regular AE assessments, vital signs, 12-lead electrocardiograms (ECGs), clinical laboratory assessments, and safety electroencephalograms (EEGs) conducted in a resting state with eyes open and closed, with photic stimulation, and with 3 minutes of hyperventilation. In addition, the patient trial included frequent suicidality assessment using the Beck Scale for Suicidal Ideation (BSS). ${ }^{[32]}$

In study 1 , the MTD was not specifically defined a priori; however, safety and tolerability were closely monitored by the study investigators and the sponsor in a blinded fashion, and dosing progression was largely dependent on absence of medication discontinuations due to AEs. The MTD in study 2 was defined a priori as the dose one step below the minimum intolerated dose (MID), where the MID was the dose at which $\geq 50 \%$ of subjects experienced multiple moderate AEs or a single severe AE, or the dose at which a serious AE occurred in one or more subjects. Safety/tolerability data were reviewed by the study investigators and the sponsor on an interim and blinded basis before progression to the next dosing level/cohort. 


\section{Pharmacokinetic Assessments}

Pharmacokinetic assessments were performed following a rich pharmacokinetic sampling scheme in both studies. In study 1, pharmacokinetic samples were taken at pre-dose, at 5, 10, 15, 30, and 45 minutes, and at 1, 1.5, 2, 2.5, 3, 4, 6, 8, 12, 18, $24,36,48$, and 72 hours post-dose upon singledose administration during part I and upon the first (no 36-, 48-, or 72-hour samples) and final dose (no 72-hour sample) in group 3 during part II of this study. For group 4 during part II, an identical sampling scheme was applied up to 12 hours post-dose on days $2(100 \mathrm{mg}), 8(225 \mathrm{mg})$, $11(325 \mathrm{mg})$, and $14(400 \mathrm{mg})$, while additional pharmacokinetic samples at 18 and 24 hours postdose were taken 18 and 24 hours after the final dose. Pharmacokinetic assessments up to 4 hours post-dose were performed under fasted conditions, with the exception of group 3, where on days 5 and 6 the food effect (a high-fat breakfast) on the pharmacokinetics of Org 26576 was specifically investigated. In study 2 , plasma pharmacokinetic samples were taken at pre-dose, at 15, 30, and 45 minutes, and at 1, 1.5, 2, 3, 4, 6, 8, and 12 hours post-dose (but before the evening dose) within a multiple-dosing scheme. To examine the extent to which Org 26576 is able to cross the human bloodbrain barrier, continuous CSF was collected over intervals of 30 minutes, starting 2 hours prior to the morning dose through 12 hours following the morning dose on day 1 and day 10 in cohort D only $(n=6)$. In this study, patients were required to eat a light breakfast 30 minutes before the morning dose.

\section{Study Medication}

In Study 1, Org 26576 was provided as freezedried cake and was reconstituted at the site pharmacy in $10 \mathrm{~mL}$ of sterile water and added to a gelatin/mannitol solution in order to obtain a final volume of $50 \mathrm{~mL}$. Placebo was composed of $50 \mathrm{~mL}$ of the gelatin/mannitol solution. The required dose was administered as an oral solution. In Study 2, Org 26576 and placebo were prepared as indistinguishable capsules containing placebo, $50 \mathrm{mg}$, or $100 \mathrm{mg}$ of Org 26576 for oral administration. The change of medication from oral solution to capsule was not expected to lead to significant formulation-dependent differences in the overall disposition of the drug. This assumption was supported by the overall physicochemical characteristics (Biopharmaceutica Classification System [BCS] class I ${ }^{[33]}$ and the in vitro absorption, distribution, metabolism, and excretion (ADME) profile of Org 26576 (Merck Sharp \& Dohme Corp., unpublished data).

\section{Bioanalysis}

Org 26576 and its internal standard were isolated from ethylene diamine tetra-acetic acid (EDTA) plasma by solid-phase extraction. Liquid chromatography-mass spectrometry using turbo electrospray ionization in multi-reaction monitoring mode was performed in order to quantify the concentration of Org 26576. The assay has been validated over the range of $0.2-200 \mathrm{ng} / \mathrm{mL}$. Both trials utilized the same bioanalytic approach and followed the same standard operating procedures. Laboratory assessments were conducted in full compliance with Good Laboratory Practice.

\section{Statistical Analysis}

\section{Safety and Tolerability}

All endpoints for both studies were analyzed using the all-subjects-treated population (participants who took at least one dose of the study medication). Descriptive statistics were calculated; however, no statistical testing was performed.

\section{Pharmacokinetics}

In study 1, group 3, a one-way analysis of variance (ANOVA) with a 'Food' factor and repeated measures for each subject on food was applied to the parameters pertaining to treatment with and without food (i.e. the maximum plasma drug concentration $\left[\mathrm{C}_{\max }\right]$ during a dosage interval at steady state $\left[\mathrm{C}_{\max , \text { ss }}\right]$, the time to reach $\mathrm{C}_{\max }$ $\left[\mathrm{t}_{\text {max }}\right]$ following drug administration at steady state $\left[\mathrm{t}_{\max , \mathrm{ss}}\right]$, and the area under the plasma concentration-time curve [AUC] from 0 to 12 hours at steady state $\left[\mathrm{AUC}_{12, \mathrm{ss}}\right]$ ), which were compared by means of a one-way ANOVA with a 'Food' factor and repeated measures for each subject on 
food (i.e. a paired t-test). Food-independent pharmacokinetics were to be concluded if the food effect for all parameters analyzed was not significant. An analogous analysis was applied to explore the presence of a regimen effect $(100 \mathrm{mg}$ single dose on day 1 , versus $100 \mathrm{mg}$ bid on day 9). For this purpose, parameters pertaining to single dosing (i.e. $\mathrm{C}_{\max }, \mathrm{t}_{\max }$, the elimination half-life $\left[t_{1 / 2}\right]$, and the AUC from time zero to infinity $\left[\mathrm{AUC}_{\infty}\right]$ ) and to steady state (i.e. $\mathrm{C}_{\text {max,ss }}, \mathrm{t}_{\mathrm{max}, \mathrm{ss}}$, $\mathrm{t}_{1 / 2, \mathrm{ss}}$, and $\left.\mathrm{AUC}_{\infty, \mathrm{ss}}\right)$ were compared. Timeindependent pharmacokinetics were to be concluded if the regimen effect for all parameters analyzed was not significant. In group 4, the parameters (i.e. the dose-normalized [dn] $\mathrm{C}_{\text {max,ss }}$ $\left[\mathrm{dn}-\mathrm{C}_{\text {max,ss }}\right], \mathrm{t}_{\mathrm{max}, \mathrm{ss}}$, and $\left.\mathrm{dn}-\mathrm{AUC}_{12, \mathrm{ss}}\right)$ pertaining to the escalating multiple dosing $(100,225,325$, and $400 \mathrm{mg}$ ) were compared by means of a twoway ANOVA with fixed factors of 'Dose' and 'Subject'. If there was no significant dose effect, then the pharmacokinetics were to be considered as linear within the dose range studied. In study 2 , the Org 26576 pharmacokinetic parameters in part II (i.e. dn- $\mathrm{C}_{\max }, \mathrm{t}_{1 / 2}, \mathrm{dn}$-AUC, and $\mathrm{t}_{\max }$ ) were compared by means of an ANOVA with fixed factors of 'Dose' (low dose: Org $26576100 \mathrm{mg}$ bid group; high dose: Org $26576400 \mathrm{mg}$ bid group), 'Day' $(1,4,27)$, 'Dose*Day' interaction, and repeated measures for each subject (subject nested within dose).

In both studies, $\log _{\mathrm{e}}$-transformed values of the parameters were used in the ANOVAs, except for $\mathrm{t}_{\max }$, for which a Wilcoxon signed rank test was performed. Effects were considered as significant if the $p$-value was $\leq 0.05$ ( $p$ : two-sided tail probability). Since these studies were not powered, all p-values were to be interpreted in the perspective of the explorative character of these trials. The plots, parameters, and analysis pertaining to the pharmacokinetic evaluations were generated using SAS release 9.1 under the Windows XP operating system.

\section{Results}

\section{Baseline Characteristics}

A total of 36 and 54 subjects were randomized in the $\mathrm{HV}$ and patient studies, respectively (see table I). All received at least one dose of study medication and therefore qualified for the allsubjects-treated population. All participants in the HV study were male, as were the majority $(65 \%)$ in the patient study. The patient study sample was more racially diverse and somewhat older than the HV sample. Weight and body mass index (BMI) were comparable between studies. In the patient study, the majority of patients $(76 \%)$ were diagnosed with recurrent depression, and the number of lifetime episodes was 4.4; the mean QIDS-C total score at baseline was 15.1 (standard deviation [SD] 2.26), reflecting moderately severe depression. ${ }^{[31]}$

\section{Safety and Tolerability}

\section{Study 1}

There were no serious or severe AEs in this study. In study part I (single dose), no subjects discontinued because of AEs. Doses of $100 \mathrm{mg}$ and higher were associated with gastrointestinal AEs (nausea, vomiting, once at 200 and $250 \mathrm{mg}$ ) and CNS AEs (dizziness, postural dizziness, headache, and paraesthesia). The $100 \mathrm{mg}$ dose was determined to be the single-dose MTD.

In part II of the study (multiple dose), Org $26576100 \mathrm{mg}$ bid given for 7 days (group 3) was well tolerated by all subjects. The AEs reported most frequently in the active-treatment group included mild dizziness and mild nausea. In group 4, where an up-titration schedule to $400 \mathrm{mg}$ bid was applied, Org 26576 was tolerated up to doses of $225 \mathrm{mg}$ bid. However, at higher doses, three subjects discontinued, two because of nausea and/or vomiting (both at $325 \mathrm{mg}$ bid) and one because of dizziness (at $400 \mathrm{mg}$ bid). The most common treatment-emergent AEs associated with Org 26576 (occurring in $\geq 25 \%$ of subjects in the activetreatment group of any study group, and with at least $2 \times$ the incidence in the placebo group) were nausea, dizziness, and somnolence, as well as feeling drunk and postural dizziness. The MTD with titration was determined to be $225 \mathrm{mg}$ bid.

There were no obvious treatment-related changes observed either for individual subjects or in the summary data for clinical laboratory values, vital signs, or ECG measurements. During the dose- 
Table I. Demographic and baseline characteristics of randomized subjects

\begin{tabular}{|c|c|c|}
\hline Characteristic & $\begin{array}{l}\text { Study 1: } \\
\text { Org 26576 in HVs } \\
(n=36)\end{array}$ & $\begin{array}{l}\text { Study 2: } \\
\text { Org } 26576 \text { in MDD } \\
\text { patients }(n=54)\end{array}$ \\
\hline \multicolumn{3}{|l|}{ Treatment assignment (n [\%]) } \\
\hline Active & 30 [83.3] & 36 [66.7] \\
\hline Placebo & $6[16.7]^{\mathrm{a}}$ & 18 [33.3] \\
\hline \multicolumn{3}{|l|}{$\operatorname{Sex}(n[\%])$} \\
\hline Male & $18[100]$ & 35 [64.8] \\
\hline Female & 0 & 19 [35.2] \\
\hline \multicolumn{3}{|l|}{ Race (n [\%]) } \\
\hline Asian & $1[2.8]$ & $3[5.6]$ \\
\hline Black & $3[8.3]$ & 14 [25.9] \\
\hline White & $29[80.6]$ & $33[61.1]$ \\
\hline Other & $1[2.8]$ & $4[7.4]$ \\
\hline Age (y; mean [range]) & $27.6[19-41]$ & $35.4[18-61]$ \\
\hline Weight (kg; mean [range]) & $77.1[66.5-94.3]$ & $76.9[44.0-118.1]$ \\
\hline Body mass index $\left(\mathrm{kg} / \mathrm{m}^{2} ;\right.$ mean [range] $)$ & $24.4[22.0-28.0]$ & $25.3[19.0-31.9]$ \\
\hline Baseline QIDS-C score (mean [SD]) & NA & $15.1[2.26]$ \\
\hline \multicolumn{3}{|l|}{ Course of depression } \\
\hline First episode (n [\%]) & NA & $13[24]$ \\
\hline Recurrent (n [\%]) & NA & $41[76]$ \\
\hline Total number of lifetime episodes (mean [range]) & NA & $4.4[1-20]$ \\
\hline \multicolumn{3}{|c|}{$\begin{array}{l}\text { Accounts for those subjects who received placebo only (i.e. subjects who were part of the active/treatment crossover design of part I are not } \\
\text { counted in the placebo total). }\end{array}$} \\
\hline \multicolumn{3}{|c|}{$\begin{array}{l}\mathbf{H V}=\text { healthy volunteer; } \mathbf{M D D}=\text { major depressive disorder; } \mathbf{N A}=\text { not applicable; QIDS-C= Quick Inventory of Depression Symptomatology - } \\
\text { Clinician Rating; } \mathbf{S D}=\text { standard deviation. }\end{array}$} \\
\hline
\end{tabular}

titration part of the study, five of six subjects taking Org 26576, but no placebo subjects, had EEG observations that were interpreted as nonspecific and indicative of drowsiness.

\section{Study 2}

No randomized patients experienced a serious or severe AE. Two subjects discontinued the trial because of AEs: one subject taking Org 26576 in part I discontinued because of akathisia at the highest dose (600 $\mathrm{mg}$ bid), and one subject taking placebo in part II discontinued because of tension headache. Most subjects in the active-treatment and placebo groups reported at least one $\mathrm{AE}$ during the treatment period (Org 26576: 97\%; placebo: $89 \%$ ). The treatment-emergent AEs reported most frequently in the active-treatment group ( $\geq 25 \%$ of subjects in either study part and with at least $2 \times$ the incidence in the placebo group) were insomnia, dizziness, nausea, muscle twitch- ing, fatigue, and feeling drunk (described by the investigator as a subjective feeling of 'fuzzy headedness' without objective impairment). On the basis of a post-study unblinded data review, it was determined that in cohort $\mathrm{C}$, two of four drugtreated subjects experienced multiple moderate AEs at the $600 \mathrm{mg}$ bid dose level. In addition, the only active-treatment discontinuation - and, regardless of titration schedule, the majority of moderate AEs - occurred at the dose of $600 \mathrm{mg}$ bid. Therefore, the MTD for this study was considered to be $450 \mathrm{mg}$ bid. The optimal starting dose was determined to be $200 \mathrm{mg}$ bid on the basis of the finding that the initial dose of $300 \mathrm{mg}$ bid was associated with more treatment-related AEs than the initial dose of 100 or $200 \mathrm{mg}$ bid.

There were no clinically significant drug-related laboratory, vital sign, ECG, or EEG findings in the study. Orthostatic tachycardia and orthostatic hypotension occurred at higher rates in the 
Table II. Pharmacokinetic parameters in group 3 healthy volunteers in study $1^{\mathrm{a}}$

\begin{tabular}{|c|c|c|c|c|}
\hline \multirow[t]{2}{*}{ Parameter } & \multirow{2}{*}{$\begin{array}{l}100 \mathrm{mg} \text { single dose } \\
\text { Day } 1 \text { fasted } \\
(\mathrm{n}=6)\end{array}$} & \multicolumn{3}{|l|}{$100 \mathrm{mg}$ bid } \\
\hline & & $\begin{array}{l}\text { Day } 9 \text { fasted } \\
(n=6)\end{array}$ & $\begin{array}{l}\text { Days } 5-6 \text { fasted } \\
(n=6)\end{array}$ & $\begin{array}{l}\text { Days } 5-6 \text { fed } \\
(n=6)\end{array}$ \\
\hline $\mathrm{dn}-\mathrm{C}_{\max }(\mathrm{ng} / \mathrm{mL} / \mathrm{mg})$ & $16.3[22.8]$ & $15.7[34.8]$ & $14.2[20.9]$ & $7.01[19.4]$ \\
\hline$t_{\max }(h ;$ median [range]) & $0.50[0.25-0.75]$ & $0.50[0.50-1.03]$ & $0.75[0.50-1.00]$ & $1.25[0.25-4.00]$ \\
\hline$t_{1 / 2}(h)$ & $2.94[14.5]$ & $2.8[24.8]$ & NA & NA \\
\hline $\mathrm{dn}-\mathrm{AUC} \mathrm{C}_{\infty}(\mathrm{ng} \bullet \mathrm{h} / \mathrm{mL} / \mathrm{mg})$ & $52.0[40.2]$ & $58.0[47.7]$ & NA & NA \\
\hline $\mathrm{dn}-\mathrm{AUC}_{12}(\mathrm{ng} \bullet \mathrm{h} / \mathrm{mL} / \mathrm{mg})$ & NA & NA & $49.0[38.4]$ & $43.0[37.1]$ \\
\hline $\mathrm{CL} / \mathrm{F}(\mathrm{L} / \mathrm{h})$ & $21.2[28.8]$ & $19.7[32.2]$ & $22.3[27.6]$ & 25.4 [28.3] \\
\hline$\underline{\mathrm{V}_{\mathrm{z}} / \mathrm{F}(\mathrm{L})}$ & $87.2[21.7]$ & $75.6[21.1]$ & NA & NA \\
\hline
\end{tabular}

a The values are expressed as arithmetic mean [CV\%] unless specified otherwise.

$\mathbf{A U C}=$ area under the plasma concentration-time curve; $\mathbf{A U C}_{\mathbf{1 2}}=\mathrm{AUC}$ from 0 to 12 hours; $\mathbf{A} \mathbf{U C}_{\infty}=\mathrm{AUC}$ from time zero to infinity; bid $=$ twice daily; $\mathbf{C L} / \mathbf{F}=$ apparent total drug clearance after oral administration; $\mathbf{C}_{\max }=$ maximum observed plasma drug concentration; $\mathbf{C V}=\mathbf{C o e f f i c i e n t}$ of variation; $\mathbf{d n}=$ dose-normalized; $\mathbf{N A}=$ not applicable; $\mathbf{t}_{1 / 2}=$ elimination half-life; $\mathbf{t}_{\max }=$ time to $\mathrm{C}_{\max } ; \mathbf{V}_{\mathbf{z}} / \mathbf{F}=$ apparent volume of distribution during the terminal phase after oral administration.

drug-treated groups than in the placebo group, though the findings were not considered clinically significant by the investigator and were not associated with any clinical signs. Nine subjects taking active medication (in contrast with zero placebo-treated subjects) had abnormal intreatment EEG observations, which were felt by the investigator to be not clinically significant, primarily associated with drowsiness, and not indicative of pro-epileptic properties of the drug. No notable differences were observed between treatment groups in the baseline-to-endpoint suicidality mean scores (as measured by the BSS).

\section{Pharmacokinetics}

As one aim of the current paper is to compare the pharmacokinetic properties of Org 26576 in two different populations, the pharmacokinetic results reported here focus on the results obtained from both studies for identical doses administered in comparable multiple-dose regimens. Food and regimen analysis results for HVs, as well as dose and regimen results for MDD patients, are presented to further elucidate the overall pharmacokinetic profile of Org 26576.

\section{Study 1: Food, Regimen, and Dose Effects}

After oral administration, Org 26576 was rapidly absorbed as well as eliminated (see table II). Plasma concentrations reached $\mathrm{C}_{\max }$ values about half an hour post-dose and quickly decayed, with a $t_{1 / 2}$ of about 3 hours. These pharmacokinetic characteristics were consistently observed upon singledose and multiple-dose administration of Org $26576100 \mathrm{mg}$ as long as the volunteers were fasted. Intake of a high-fat breakfast prior to dosing affected the pharmacokinetic characteristics of Org 26576 by increasing $\mathrm{t}_{\max }$ by about $40 \%$ and by reducing $\mathrm{C}_{\max }$ by approximately $50 \%$. AUC was reduced by only $12 \%$ with food, which is within the estimated variability of the parameter. ${ }^{[34]}$ This fed-state reduction in the absorption rate translated into lower and smoothed plasma concentrations around the $\mathrm{C}_{\max }$ values observed in fasted conditions. Regimen effect testing on the $\log _{\mathrm{e}}$-transformed pharmacokinetic parameters of Org 26576 showed that no significant regimen effects on $\mathrm{C}_{\max }$, total exposure, or $\mathrm{t}_{1 / 2}$ were found. Analogously, the Wilcoxon signed rank test indicated no regimen effect on $t_{\max }$.

The dose-normalized mean curves for all escalating doses in group 4 are displayed in figure 2, and the descriptive statistics for key pharmacokinetic parameters of the $100 \mathrm{mg}$ and $400 \mathrm{mg}$ bid escalating doses in group 4 are shown in table III. $\mathrm{C}_{\max }$ values increased subproportionally with dose, while $t_{\max }$ and AUC values showed the opposite trend. When compared with the results in group 3 , the $t_{1 / 2}$ was not clearly affected by the dose. 


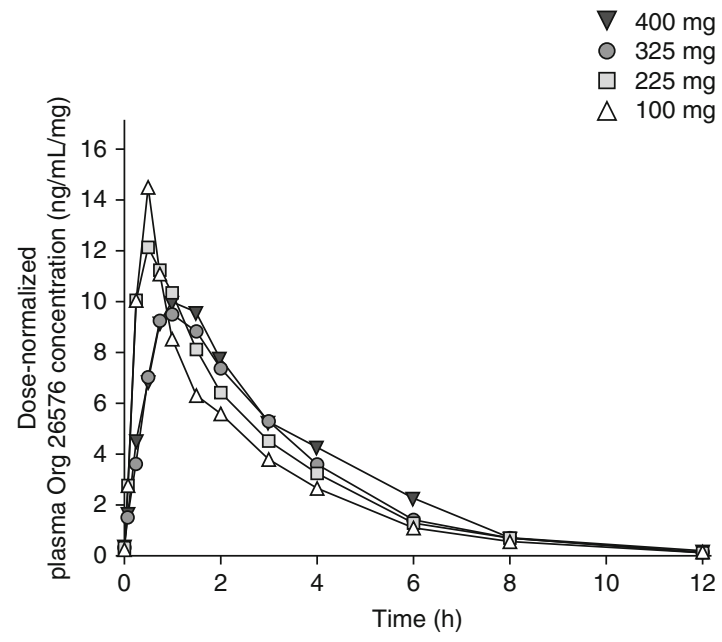

Fig. 2. Mean dose-normalized plasma concentrations of escalating doses of Org 26576 in healthy volunteers.

An overall trend for the dose effect was found for $\mathrm{dn}-\mathrm{C}_{\text {max,ss }}(\mathrm{p}=0.09)$ and was significant for $\mathrm{dn}-\mathrm{AUC}_{12, \mathrm{ss}}(\mathrm{p}=0.03)$. The ANOVA on ranks of $t_{\max }$ resulted in a significant dose effect, showing larger $t_{\max }$ values for the highest doses (325 and $400 \mathrm{mg})$ than for the lower doses (100 and $225 \mathrm{mg}$ ).

\section{Study 2: Dose and Day Effects}

The mean dose-normalized plasma concentrations observed in part II of this study at days 1 , 4 , and 27 for the $100 \mathrm{mg}$ and $400 \mathrm{mg}$ bid treat- ment groups are displayed in figure $3 \mathrm{a}$ and $3 \mathrm{~b}$, respectively. The mean $\mathrm{dn}-\mathrm{C}_{\max }$ and $\mathrm{dn}$-AUC values for days 4 and 27 in the $100 \mathrm{mg}$ bid treatment group (see table III) were approximately $30 \%$ higher than for day 1 (data not shown). For the $400 \mathrm{mg}$ bid treatment group, similar mean dn$\mathrm{C}_{\max }$ and $\mathrm{dn}$-AUC values were found for all days. The mean dose-normalized exposure values for the $400 \mathrm{mg}$ bid group tended to be somewhat higher than those for the $100 \mathrm{mg}$ bid group (see table III). An explorative ANOVA on all subjects in part II showed no statistically significant overall 'Dose', 'Day', or 'Dose*Day' effects on dn$\mathrm{C}_{\max }, \mathrm{t}_{\max }, \mathrm{dn}$-AUC, and $\mathrm{t}_{1 / 2}$. No major deviations from the dose proportionality and time independence of the kinetics of Org 26576 were observed in this study in the titration schemes and dose range tested. For cohort D of part I, the mean Org 26576 exposure and concentration values in plasma and CSF were similar, both on day 1 (100 mg single dose) and on day 10 (300 mg steady state). The CSF $t_{\max }$ followed the plasma $t_{\max }$ by approximately 15 minutes with single doses and by approximately 1.5 hours with multiple doses.

\section{Discussion and Conclusion}

The studies presented herein describe bridging data for the AMPA PAM Org 26576. On the basis of evidence suggesting that neuropsychiatric patients often tolerate higher medication

Table III. Pharmacokinetic parameters in healthy volunteers in study 1 and in patients with major depressive disorder in study $2^{\mathrm{a}}$

\begin{tabular}{|c|c|c|c|c|c|c|}
\hline \multirow[t]{4}{*}{ Parameter } & \multicolumn{2}{|l|}{ Study 1 in HVs } & \multicolumn{4}{|c|}{ Study 2 in MDD patients } \\
\hline & \multirow{3}{*}{$\begin{array}{l}100 \mathrm{mg} \text { bid } \\
\text { Day } 2 \\
(\mathrm{n}=6)\end{array}$} & \multirow{3}{*}{$\begin{array}{l}400 \mathrm{mg} \text { bid } \\
\text { Day } 14 \\
(\mathrm{n}=3)\end{array}$} & \multicolumn{2}{|c|}{$100 \mathrm{mg}$ bid } & \multicolumn{2}{|l|}{$400 \mathrm{mg}$ bid } \\
\hline & & & Day 4 & Day 27 & Day 4 & Day 27 \\
\hline & & & $(n=10)$ & $(n=10)$ & $(n=10)$ & $(n=9)$ \\
\hline$\overline{\mathrm{dn}-\mathrm{C}_{\max }(\mathrm{ng} / \mathrm{mL} / \mathrm{mg})}$ & 16.3 [38.3] & $11.2[25.8]$ & 11.4 [46.3] & $11.2[34.1]$ & $11.3[30.8]$ & $12.3[57.4]$ \\
\hline $\mathrm{t}_{\max }(\mathrm{h} ;$ median [range] $)$ & $0.5[0.25-0.50]$ & $1.00[0.75-1.50]$ & $1.75[0.75-2.13]$ & $1.50[0.75-2.00]$ & $1.75[0.75-4.0]$ & $2.00[0.75-4.00]$ \\
\hline$t_{1 / 2}(h)$ & NA & $2.40[20.4]$ & $2.1[31.4]$ & $2.3[29.9]$ & $2.49[33.0]$ & $2.47[37.6]$ \\
\hline $\mathrm{dn}-\mathrm{AUC}_{12}(\mathrm{ng} \bullet \mathrm{h} / \mathrm{mL} / \mathrm{mg})$ & $34.5[44.1]$ & $38.9[25.4]$ & $49.1[59.1]$ & $48.4[56.7]$ & $56.6[48.9]$ & $61.7[64.3]$ \\
\hline $\mathrm{CL} / \mathrm{F}(\mathrm{L} / \mathrm{h})$ & $33.6[40.4]$ & $27.0[28.9]$ & NA & $27.2[53.1]$ & NA & 35.5 [129] \\
\hline $\mathrm{V}_{\mathrm{z}} / \mathrm{F}(\mathrm{L})$ & NA & $93.6[34.1]$ & NA & $79.0[34.1]$ & NA & $112[105]$ \\
\hline
\end{tabular}

a The values are expressed as arithmetic mean [CV\%] unless specified otherwise.

$\mathbf{A U C}_{\mathbf{1 2}}=$ area under the plasma concentration-time curve from 0 to 12 hours; $\mathbf{b i d}=$ twice daily; $\mathbf{C L} / \mathbf{F}=$ apparent total drug clearance after oral administration; $\mathbf{C}_{\max }=$ maximum observed plasma drug concentration; $\mathbf{C V}=$ coefficient of variation; $\mathbf{d n}=$ dose-normalized; $\mathbf{H V}=$ healthy volunteer; $\mathbf{M D D}=$ major depressive disorder; $\mathbf{N A}=$ not applicable; $\mathbf{t}_{1 / 2}=$ elimination half-life; $\mathbf{t}_{\mathbf{m a x}}=$ time to $\mathrm{C}_{\mathrm{max}} ; \mathbf{V}_{\mathbf{z}} / \mathbf{F}=$ apparent volume of distribution during the terminal phase after oral administration. 


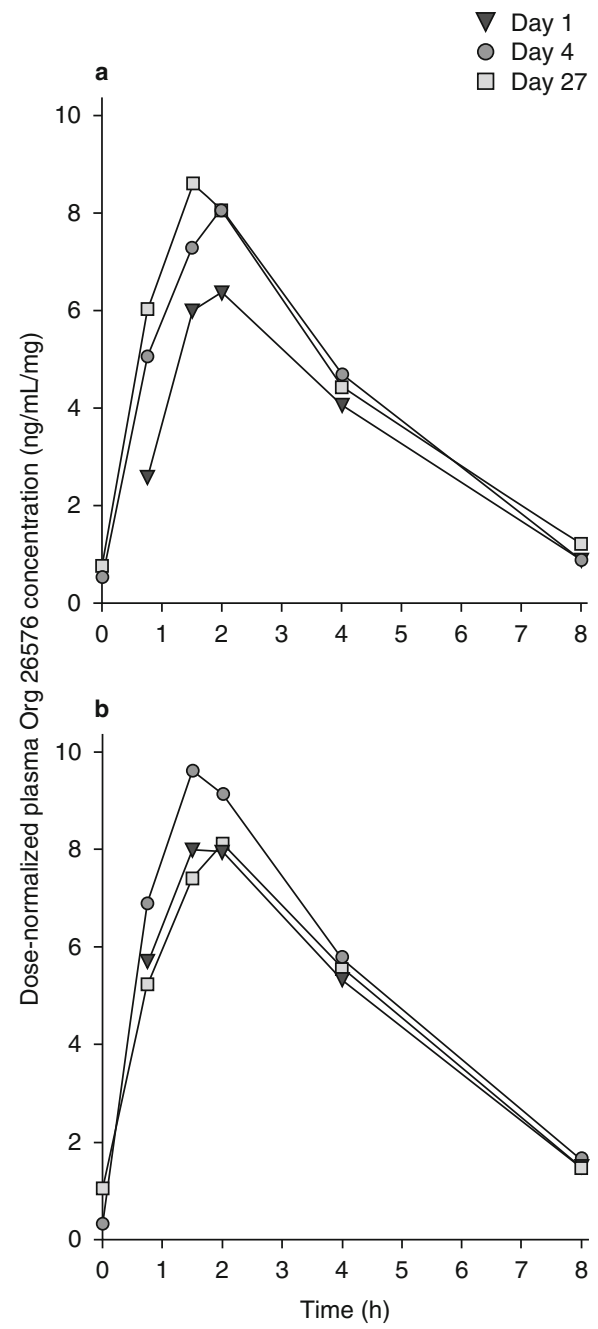

Fig. 3. Mean dose-normalized plasma concentrations of Org 26576 on days 1,4 , and 27 for (a) the $100 \mathrm{mg}$ twice-daily dose and (b) the $400 \mathrm{mg}$ twice-daily dose in MDD patients.

doses than do HVs, the clinical development plan for the Org 26576 program included both phase I (HVs) and phase Ib (patients diagnosed with MDD) multiple-rising-dose studies. The primary objectives were to establish the MTD and to fully characterize the safety, tolerability, and pharmacokinetics in both populations. Although the trials differed in several design elements, we believe that the data presented here are both comparable and interpretable, given that they are based on trial cohorts that included the same multiple-rising-dose approach, starting dose, and regimen; nearly identical titration steps; similar housing conditions; and a similar safety assessment strategy.

In the HV trial, Org 26576 was well tolerated at doses of up to $225 \mathrm{mg}$ bid, while in depressed patients, the MTD was $450 \mathrm{mg}$ bid - twice the maximum dose established in HVs. The patient trial also established that slightly faster titration could be achieved without increasing the number of dose-limiting AEs. The most common AEs associated with the study drug in both populations included dizziness, nausea, and feeling drunk. There were no clinically relevant safety issues associated with Org 26576 at any dose in either population.

In an attempt to learn whether better tolerability in patients could be explained by pharmacokinetic differences between populations, we examined pharmacokinetic parameters for both HVs and patients under highly comparable dosing conditions. Multiple-dose administration of Org 26576 at the same dose level in HVs and MDD patients resulted in pharmacokinetic profiles that were similar overall, though not identical. In both populations, Org 26576 was rapidly absorbed and disposed, with a $t_{1 / 2}$ not longer than 3 hours. $\mathrm{C}_{\max }$ and $\mathrm{t}_{\max }$ values increased subproportionally and underwent a time delay, suggesting a dose-dependent, partially saturated absorption process, although not statistically significant. Further, no regimen effects were observed, indicating linear kinetics over time. The overall exposure of the drug, however, seemed to be somewhat higher and $t_{\max }$ values seemed to be greater in patients than in HVs. While the origin of the exposure difference is unclear, food and formulation effects cannot be entirely excluded as underlying causes of the $t_{\max }$ difference.

Indeed, one of the principal limitations in this population comparison is the difference between studies under fed/fasted conditions. As is generally the case with early clinical drug development, the phase I and Ib studies were conducted in succession and were designed to build on accumulating animal and human data and other program advancements. The HV study finding of 
a food effect indicated that $\mathrm{C}_{\max }$ values were higher in the fasted state than in the fed state, suggesting that the $\mathrm{C}_{\max }$ 'smoothing' effect of food intake prior to dosing could reduce the risk and/or the frequency of peak-related AEs. Under this assumption, the patient study protocol required all doses to be administered 30 minutes after a meal. However, we do not believe that the food effect on pharmacokinetics fully explains the higher MTD in depressed patients. $\mathrm{C}_{\max }$ values were comparable between populations at the higher doses, which were the points at which dose-limiting AEs occurred, and the events that drove MTD determination in both studies were not often associated with $t_{\max }$.

Another evolutionary program change was the inclusion of females midway through the patient trial following the finalization of animal reprotoxicity studies. While the HV study included only males, $36 \%$ of treated participants in the patient study were female. Although this change was necessary in order to examine safety and tolerability in the broader target population, it raises the question as to whether tolerability differences between the trials can be attributed to sex. However, post hoc evaluation showed that exclusion of female subjects from the patient sample did not change the MTD determination at all.

An important difference between trials is how the MTD was defined. In the HV study, the MTD was driven by discontinuations due to AEs. In the patient study, the MTD was defined a priori as the dose one step below the MID, where the MID was the dose at which $\geq 50 \%$ of subjects experienced multiple moderate AEs or a single severe $\mathrm{AE}$, or the dose at which a serious AE occurred in one or more subjects. If we applied the HV approach to the patient study, the MTD result would not change. In contrast, if we applied the patient definition to the HV study, an MTD would not be defined, because only one patient experienced multiple moderate AEs. However, we note that patients were much more likely than HVs to continue dosing despite moderate-intensity events. In the HV trial, every subject who reported a moderate AE ultimately discontinued treatment because of the event. In contrast, only one par- ticipant of nine who experienced moderate AEs in the patient trial discontinued. Whether this is due to better tolerability in general, greater motivation to stay in the treatment unit for lifestyle reasons, the possibility of a treatment effect, differences in the clinical approaches used by different sites and investigators, or some other factor, is difficult to determine. Regardless, the MTD determinations reflect the experience of the participants and the clinical impressions of the investigators, suggesting that the underlying definitions were appropriate for the populations under study.

Although we certainly acknowledge the scientific value of clearly defining the MTD in advance of study implementation, we believe, on the basis of this experience, that clinical judgment has an equally important role. Given the very small sample size included in most tolerability studies, under strict a priori criteria small numbers of AEs can drive the MTD determination. When AEs are of questionable relationship to the study drug or are reported by unreliable patients - or, conversely, when safety issues are seen that do not easily fit the MTD criteria - rigid adherence to an a priori definition could result in inappropriate dose selection for phase II trials. For this reason, the current phase Ib protocol included provisions for independent unblinded data review if needed to elucidate the tolerability profile, as well as flexibility to allow clinical judgment in the final determination of the MTD.

Whether better patient tolerability can be attributed in this case to alteration of receptor activity by previous antidepressant treatment is an open question. Currently marketed antidepressants are thought to have eventual downstream effects on the glutamate system ${ }^{[35]}$ and on AMPA receptors themselves, ${ }^{[36]}$ suggesting that a prior treatment history could influence tolerability even with this novel compound. However, we note that in the current trial, patients presenting with their first episode of depression (with no prior antidepressant taken in that episode) and those presenting with recurrent depression (and presumably a more robust treatment history) demonstrated very comparable tolerability profiles. Alternative explanations include the possibility 
that alteration of receptor activity by depression itself drives better tolerability in patients. Indeed, there is a growing body of evidence suggesting that both glutamate activity ${ }^{[22-24]}$ and AMPA receptor expression ${ }^{[36]}$ are altered in depressed patients. However, the mechanism by which these findings translate into decreased glutamate drug sensitivity remains to be explored.

As a result of this detailed bridging work and further information from animal and human pharmacokinetic/pharmacodynamics modeling, which predicted target levels of AMPA receptor engagement at doses ranging from 100 to $400 \mathrm{mg}$ bid, ${ }^{[37]}$ the upper end of the dose range selected for phase II efficacy trials was significantly higher than the HV MTD. Final dose selection also took into consideration the likelihood that patient tolerability could differ in an outpatient setting, where life demands may mediate the functional impairment associated with drug-related AEs. Here too, patient tolerability data helped to address this question by providing critical information regarding the time of onset, severity, and duration of AEs, and the tendency for specific events to abate over time. The Org 26576 bridging data therefore contributed to confident dose selection for phase II trial planning and, as a result, served the greater purpose of patient and program risk minimization.

\section{Acknowledgments}

Drs. Nations, Bursi, Dogterom, Ereshefsky, Gertsik, Mant, and Schipper are responsible for the work described in this paper. All authors were involved in at least one of the following: conception, design, data acquisition, data analysis, statistical analysis, and interpretation of data. All authors drafted the manuscript and/or revised the manuscript for important intellectual content, and all authors provided final approval of the version to be published.

Organon (now Merck \& Co., Inc.) provided the study drug (Org 26576) and financial support for the conduct of the studies.

Dr. Nations was employed by Merck Sharp \& Dohme Corp. (Whitehouse Station, NJ, USA) at the time of this research. Drs. Bursi and Schipper were employed by Merck Sharp \& Dohme Oss BV (Oss, the Netherlands) at the time of this research. Dr. Dogterom is currently an employee of Merck Sharp \& Dohme Oss BV. The employers of Drs. Ereshefsky and Gertsik (California Clinical Trials Medical Group, Inc.) and Dr. Mant (Quintiles) were paid by Organon (now Merck \& Co. Inc.) for their work on this trial.

\section{References}

1. Cutler NR, Sramek JJ, Murphy MF, et al. Critical pathways to success in CNS drug development. 1st ed. Oxford: Wiley-Blackwell, 2010

2. Sramek JJ, Cutler NR. Investigator perspective on MTD: practical application of an MTD definition - has it accelerated development? J Clin Pharmacol 2000; 40: 1184-7

3. Ereshefsky L, Jhee S, Gertsik L, et al. Strategies to accelerate drug development for CNS compounds: focus on schizophrenia [poster]. 15th Biennial Winter Workshop in Psychoses; 2009 Nov 15-18; Barcelona

4. Vanover K, Davis R, Ereshefsky L, et al. Safety, pharmacokinetics and early signals for efficacy of ITI-007, a novel investigational drug for the treatment of schizophrenia and related disorders [poster]. 13th International Congress on Schizophrenia Research; 2011 Apr 2-6; Colorado Springs (CO)

5. Cutler NJ, Sramek JJ. Guidelines for conducting bridging studies in Alzheimer's disease. Alzheimer Dis Assoc Disord 1998; 12 (2): 88-92

6. Cutler NR, Sramek JJ, Greenblatt DJ, et al. Defining the maximum tolerated dose: investigator, academic, industry, and regulatory perspectives. J Clin Pharmacol 1997; 37 (9): $767-83$

7. Anand R, Geffen Y, Vasile D, et al. An open-label tolerability study of BL-1020 antipsychotic: a novel gamma aminobutyric acid ester of perhenazine. Clin Neuropharmacol 2010; 33 (6): 297-302

8. Fitzgerald PB. BL-1020: an oral antipsychotic agent that reduces dopamine activity and enhances $\mathrm{GABA}_{\mathrm{A}}$ activity, for the treatment of schizophrenia. Curr Opin Investig Drugs 2010; 11 (1): 92-100

9. Ereshefsky L, Gage A, Yu B, et al. Phase 1 study of RGH188 in schizophrenic patients [poster]. 161st Annual Meeting of the American Psychiatric Association; 2008 May 3-8; Washington, DC

10. Sramek JJ, Kirkesseli S, Paccaly-Moulin A, et al. A bridging study of fananserin in schizophrenic patients. Psychopharmacol Bull 1998; 34 (4): 811-8

11. Ereshefsky L, Jhee S, Phillips D, et al. Assessment of the maximum tolerated dose (MTD) of lurasidone in patients with schizophrenia [poster]. 48th Annual Meeting of the American College of Neuropsychopharmacology; 2010 Dec 6-10; Hollywood (FL)

12. Sramek JJ, Block GA, Reines SA, et al. A multiple-dose safety trial of epastigmine in Alzheimer's disease, with pharmacodynamic observations of red blood cell cholinesterase. Life Sci 1995; 56 (5): 319-26

13. Lefevre G, Sedek G, Jhee S, et al. Pharmacokinetics and pharmacodynamics of the novel daily rivastigmine transdermal patch compared with twice-daily capsules in Alzheimer's disease patients. Clin Pharmacol Ther 2008; 83 (1): 106-14

14. Gobburu JV, Tammara V, Lesko L, et al. Pharmacokineticpharmacodynamic modeling of rivastigmine, a cholinesterase inhibitor, in patients with Alzheimer's disease. J Clin Pharmacol 2001; 41 (10): 1082-90

15. Cutler NR, Jhee SS, Cyrus P, et al. Safety and tolerability of metrifonate: results of a maximum tolerated dose study. Life Sci 1996; 62 (16): 1433-41

16. Sramek JJ, Eldon MA, Posvar EL, et al. Initial safety, tolerability, pharmacodynamics, and pharmacokinetics of 
CI-1007 in patients with schizophrenia. Psychopharm Bull 1998; 34 (1): 93-8

17. Cutler NR, Murphy MF, Nash RJ, et al. Clinical safety, tolerance, and plasma levels of the oral anticholinesterase 1,2,3,4-tetrahydro-9-aminoacridin-1-oL-maleate (HP 029) in Alzheimer's disease: preliminary findings. J Clin Pharmacol 1990; 30 (6): 556-61

18. Lynch G. Glutamate-based therapeutic approaches: ampakines. Curr Opin Pharmacol 2006; 6: 82-8

19. Arai AC, Kessler M. Pharmacology of ampakine modulators: from AMPA receptors to synapses and behaviour. Curr Drug Targets 2007; 8: 583-602

20. Deakin JFW, Slater P, Simpson MDC, et al. Frontal cortical and left temporal glutamatergic dysfunction in schizophrenia. J Neurochem 1989; 52: 1781-6

21. Kerwin R, Patel S, Meldrum B. Quantitative autoradiographic analysis of glutamate binding sites in the hippocampal formation in normal and schizophrenic brain post mortem. Neuroscience 1990; 39: 25-32

22. Mitani H, Shirayama Y, Yamada T, et al. Correlation between plasma levels of glutamate, alanine and serine with severity of depression. Prog Neuropsychopharmacol Biol Psychiatry 2006; 30: 1155-8

23. Frye MA, Tsai GE, Huggins T, et al. Low cerebrospinal fluid glutamate and glycine in refractory affective disorder. Biol Psychiatry 2006; 61: 162-6

24. Hashimoto K, Sawa A, Iyo M. Increased levels of glutamate in brains from patients with mood disorders. Biol Psychiatry $2007 ; 62: 1310-6$

25. Lauterborn JC, Lynch G, Vanderklish P, et al. Positive modulation of AMPA receptors increases neurotrophin expression by hippocampal and cortical neurons. J Neurosci 2000; 20 (1): 8-21

26. Du J, Gray NA, Falke CA, et al. Modulation of synaptic plasticity by antimanic agents: the role of AMPA glutamate receptor subunit 1 synaptic expression. J Neurosci 2004; 24 (29): 6578-89

27. Bai F, Bergeron M, Nelson DL. Chronic AMPA receptor potentiator (LY451646) treatment increases cell proliferation in adult rat hippocampus. Neuropharmacology 2003; 44: $1013-21$
28. Alt A, Nisenbaum ES, Bleakman D, et al. A role for AMPA receptors in mood disorders. Biochem Pharmacol 2006; 71: 1273-88

29. O'Neill MJ, Witkin JM. AMPA receptor potentiators: application for depression and Parkinson's disease. Curr Drug Targets 2007; 8: 603-20

30. Nations KR, Dogterom P, Bursi R, et al. Evaluation of Org 26576, an AMPA receptor positive allosteric modulator, in patients diagnosed with major depressive disorder: an exploratory, randomized, double-blind, placebo-controlled trial. J Psychopharmacol. In press

31. Rush AJ, Trivedi MH, Ibrahim HM, et al. The 16-item Quick Inventory of Depressive Symptomatology (QIDS) Clinician Rating (QIDS-C) and Self-Report (QIDS-SR): a psychometric evaluation in patients with chronic major depression. Biol Psychiatry 2003; 54: 573-83

32. Beck AT, Steer RA, Ranieri WF. Scale for suicide ideation: psychometric properties of a self-report version. J Clin Psychol 1988; 44: 499-505

33. Faassen F, Vromans H. Biowaivers for oral immediaterelease products: implications of linear pharmacokinetics. Clin Pharmacokinet 2004; 43 (15): 1117-26

34. Fleisher D, Li C, Zhou Y, et al. Drug, meal, and formulation interactions influencing drug absorption after oral administration: clinical implications. Clin Pharmacokinet 1999; 36: 233-54

35. Hashimoto K. The role of glutamate on the action of antidepressants. Prog Neuropsychopharmacol Biol Psychiatry 2011; 35: 1558-68

36. Beneyto M, Kristiansen LV, Oni-Orisan A, et al. Abnormal glutamate receptor expression in the medial temporal lobe in schizophrenia and mood disorders. Neuropsychopharmacology 2007; 32: 1888-902

37. Bursi R, Erdemli G, Campbell R, et al. Translational PKPD modelling of molecular target modulation for the AMPA receptor positive allosteric modulator Org 26576. Psychopharmacology (Berl) 2011; 218: 713-24

Correspondence: Dr. Kari Nations, University of Texas at Austin, Department of Psychology, College of Liberal Arts, 1 University Station A8000, Austin, TX 78712, USA. 